\title{
Remote control of BBB: A tale of exosomes and microRNA
}

\author{
Cell Research (2017) 27:849-850. doi:10.1038/cr.2017.71; published online 19 May 2017
}

\begin{abstract}
Exosome-mediated intercellular communication has become an emerging field of human health and diseases. A recent study published by Cell Research reports that in zebrafish neurons can remotely regulate blood-brain barrier integrity by delivering miR-132 through secretion of exosomes.
\end{abstract}

In the central nervous system (CNS), neurons, glia and vascular cells including endothelial cells, vascular smooth muscle cells and pericytes communicate with each other by rapid and direct exchange of small signaling molecules through gap junctions, multi-step signaling through receptors and channels, and exosomes, for long-range communication with distant targets [1]. In contrast to peripheral organs with highly permeable capillaries [2], brain capillaries, a site of the blood-brain barrier (BBB) in vivo, prevent entry of blood-derived toxic substances and pathogens into the brain $[3,4]$. The BBB regulates transport of oxygen, energy metabolites, nutrients and regulatory molecules from blood to brain, and clears metabolic waste products from brain into circulation [1].

Brain endothelial cells are connected by tight and adherens junctions, which forms anatomical BBB [1]. Brain endothelium expresses thousands of different transporters and receptors, and is endowed with perivascular cells - pericytes that are separated from endothelium by the basement membrane. Astrocyte end-feet cover most of the vessel wall allowing direct communications between astrocytes and pericytes, from one end, and astrocytes and endothelium, from the other end [1]. Neural microenvironment plays a key role in BBB de- velopment via Wnt and Sonic Hedgehog (Shh) signaling pathways [1]. Neuronal activity influences cerebrovascular pattern [5] and transport across the BBB of substrates such as serum insulin-like growth factor-1 [6]. However, the role of exosomes in regulating BBB integrity and functions is poorly understood.

Exosomes are $30-100 \mathrm{~nm}$ doublemembrane extracellular vesicles produced by nearly every cell type. They are generated mainly from reverse budding of multivesicular bodies, and are enriched in signaling proteins and lipids of parental cells. Exosomes play an important role in neuron-glia communication and regulation of synaptic plasticity [7]. They carry and deliver pathogenic peptides, such as amyloid- $\beta$, prion, $\alpha$-synuclein and tau [7], and play a role in communication across the BBB between periphery and CNS during systemic inflammation [8].

Exosomes also carry RNAs including microRNAs. MicroRNAs are small non-coding RNAs with $\sim 22$ nucleotides capable of silencing gene expression via the RNA-induced silencing complex. In the CNS, microRNAs have been shown to not only contribute to gene regulation during development, but also to remodeling of neural circuits in adulthood. MicroRNAs have been associated with pathogenesis of many CNS disorders [9]. First known for their essential roles in cell-autonomous regulation of gene expression, the microRNA-dependent exosomal signaling is currently being recognized as an intercellular route in regulating gene expression in distant cells. For instance, in response to peripheral inflammation, exosomes from hematopoietic cells contribute to long- range RNA-based communications with Purkinje neurons in the brain across the BBB [10].

In a recent paper in Cell Research, Xu and colleagues [11] used zebrafish as a model system to examine the function of miR-132, a microRNA predominantly expressed in neurons. By antagonizing miR-132 with morpholino antisense oligonucleotides, they surprisingly found that zebrafish larvae with inactivated miR-132 (miR-132 morphants) exhibited severe intracranial hemorrhage and impaired BBB integrity, as shown by extravasation of red blood cells and brain accumulation of exogenous circulating tracers. This finding was confirmed by a genomic editing using Cas 9 (CRISPR associated protein 9) and miR-132-specific guide RNAs to disrupt the endogenous miR-132 loci (Figure 1).

Although miR-132 is enriched in neurons and known to regulate neuronal differentiation and expression of an autism-related gene $M e C P 2$ via feedback control, it is also present in endothelial cells and can regulate angiogenesis. This raises a question, which source of miR-132 is sealing the BBB? Xu and colleagues utilized a competitive inhibition approach with transcripts containing multiple miR-132-binding sites; they found that expression of miR-132 sponges using a neuron-specific $H u C$ promoter phenocopied miR-132 morphants, suggesting that the source of miR-132 is likely from neurons. The story then became more interesting, as they also found that increasing neuronal expression of $m i R-132$ in zebrafish larvae also elevated miR-132 level in endothelial cells, suggesting transfer of miR-132 from neurons to endothelial cells. Next, 


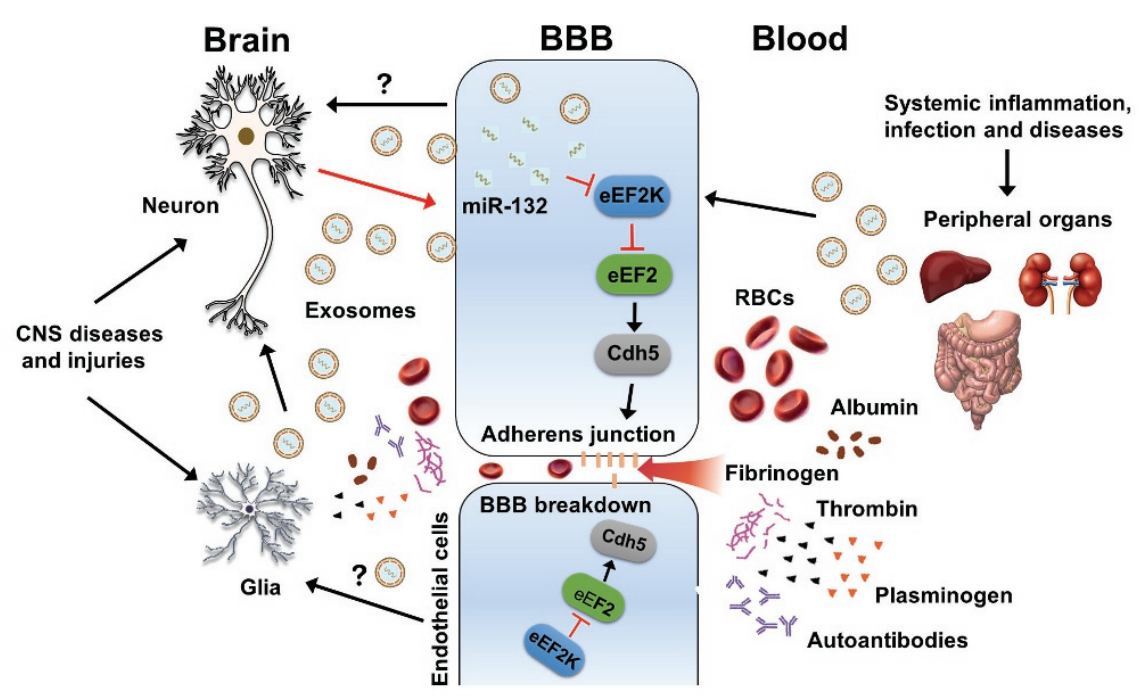

Figure 1 Regulation of BBB by micro-RNA-containing exosomes.

$\mathrm{Xu}$ and colleagues sought for direct evidence in vivo using transgenic zebrafish larvae, in which neuronal exosomes are filled with green fluorescent protein (GFP) and brain endothelial cells labeled with red fluorescent protein (RFP). They obtained time-lapse confocal images showing uptake of GFP-positive exosomes by RFP-positive endothelial cells, supporting the intercellular exosome transfer hypothesis. Additionally, they showed that purified neuronal exosomes are taken up by mouse brain endothelial cells, and exosomes secreted by mouse neurons in vitro can transfer miR-132 to co-cultured endothelial cells, suggesting that this mechanism is conserved in mammals.

What is the underlying molecular mechanism? Xu and colleagues examined multiple aspects of the BBB integrity and found that only vascular endothelial cadherin (encoded by $C d h 5$ gene), a component of the adherens junctions, was significantly downregulated in miR-132 morphants, whereas the tight junction proteins, pericyte coverage and transcytosis machinery remained unaffected. With microarray analysis of miR-132 morphants and computational prediction using MicroCosm, they also identified that $e E F 2 K$, encoding eukaryotic elongation factor 2 kinase, is the direct target of miR-132 in endothelial cells. The eEF2K inhibits translational elongation of $C d h 5$ by phosphorylation of eukaryotic elongation factor 2 (eEF2), and neuronal miR-132 releases this inhibition and allows $C d h 5$ gene expression in endothelial cells enhancing the BBB integrity.

Overall, findings by $\mathrm{Xu}$ et al. support a long-range exosome-mediated communication between neurons and brain endothelial cells, which is exciting and important. Their findings also raise a number of questions, e.g., whether brain endothelium can communicate back to neurons via endothelial-derived exosomes? And, whether the BBB in primates and humans can also be remotely controlled by miR-132? Further, future studies should explore whether greater anatomical distances in the larger human and primate brain pose a diffusional barrier for exosomal regulation of BBB permeability.

With regards to translational potential, these findings raise additional questions as to whether neuronal-derived miR-132 levels are altered in the CSF in individuals with mild cognitive impairment associated with a regional hippocampal BBB disruption [12], and/ or neurodegenerative conditions and dementia associated with small vessel disease, BBB breakdown and cerebral microhemorrhages, such as Alzheimer's disease (AD) [1]. Interestingly, the CSF microRNA profiles in $\mathrm{AD}$ and cognitively normal controls are different [13], possibly suggesting that miRNA biomarkers may add to sensitivity and specificity of AD diagnosis when combined with standard $\mathrm{AD}$ and vascular injury biomarkers. Additionally, mixing microRNAs and exosomes as nanoparticles has potential to deliver therapeutic siRNAs across the BBB into the brain. Thus, the report by $\mathrm{Xu}$ and colleagues has opened the door for a new direction and thinking in the field focused on the role of exosomes in controlling BBB integrity in health and disease.

Zhen Zhao ${ }^{1}$, Berislav V Zlokovic ${ }^{1}$

${ }^{1}$ Zilkha Neurogenetic Institute and Department of Physiology and Neuroscience, Keck School of Medicine, University of Southern California, Los Angeles, CA 90033, USA

Correspondence: Zhen Zhao, Berislav V Zlokovic ${ }^{\mathrm{b}}$

aE-mail: zzhao@usc.edu

bE-mail: zlokovic@usc.edu

\section{References}

1 Zhao Z, Nelson AR, Betsholtz C, et al. Cell 2015; 163:1064-1078.

2 Mann GE, Zlokovic BV, Yudilevich DL. Biochim Biophys Acta 1985; 819:241-248.

3 Zlokovic BV. Pharm Res 1995; 12:13951406.

4 Zlokovic BV, Apuzzo MLJ. Neurosurgery 1997; 40:789-804.

5 Lacoste B, Comin $\mathrm{CH}$, Ben-Zvi A, et al. Neuron 2014; 83:1117-1130.

6 Nishijima T, Piriz J, Duflot S, et al. Neuron 2010; 67:834-846.

7 Frühbeis C, Fröhlich D, Krämer-Albers EM. Front Physiol 2012; 3:119.

8 Balusu S, van Wonterghem E, De Rycke R, et al. EMBO Mol Med 2016; 8:1162-1183.

9 Tkach M, Théry C. Cell 2016; 164:12261232.

10 Ridder K, Keller S, Dams M, et al. PLoS Biol 2014; 12:e1001874.

11 Xu B, Zhang Y, Du XF, et al. Cell Res 2017; 27: 882-897.

12 Montagne A, Barnes SR, Sweeney MD, et al. Neuron 2015; 85:296-302.

13 Lusardi TA, Phillips JI, Wiedrick JT, et al. J Alzheimers Dis 2017; 55:1223-1233. 